\title{
The Design of Circular Microstrip Patch Antenna by Using Quasi-Newton Algorithm of ANN
}

\author{
Abhilasha Mishra', Ganesh B. Janvale ${ }^{2}$, Bhausaheb Vyankatrao Pawar ${ }^{3}$, Pradeep Mitharam Patil ${ }^{4}$ \\ ${ }^{1}$ Department of Electronics Engineering, North Maharashtra University, Jalgaon, India; ${ }^{2}$ Department of Computer Science and \\ Information Technology, Dr. Babasaheb Ambedkar Marathwada University, Aurangabad, India; ${ }^{3}$ Department of Computer Science, Mumbai \\ University, Mumbai, India; ${ }^{4}$ Department of Electronics \& Telecommunications, Vishwakarma Institute of Technology, Pune, India. \\ Email: abbhilasha@yahoo.co.in, ganeshjanvale@rediffmail.com, bvpawar@hotmail.com, patil_pm@rediffmail.com
}

Received April $1^{\text {st }}, 2010$; revised May 23 ${ }^{\text {rd }}, 2010$; accepted May 27 ${ }^{\text {th }}, 2010$.

\begin{abstract}
The paper presents the Quasi Newton model of Artificial Neural Network for design of circular microstrip antenna (MSA). In this model, a closed form expression is used for accurate determination of the resonant frequency of circular microstrip patch antenna. The calculated resonant frequency results are in good agreement with the experimental results reported elsewhere. The results show better agreement with the trained and tested data of ANN models. The results are verified by the experimental results to produce accurate ANN models. This presents ANN model practically as an alternative method to the detailed electromagnetic design of circular microstrip antenna.
\end{abstract}

Keywords: Circular Microstrip Antenna (CMSA), Artificial Neural Network (ANN), Quasi Newton (QN)

\section{Introduction}

The MSA is an excellent radiator for many applications such as mobile antenna, aircraft and ship antennas, remote sensing, missiles and satellite communications [1]. It consists of radiating elements (patches) photo etched on the dielectric substrate. Microstrip antennas are low profile conformal configurations. They are lightweight, simple and inexpensive, most suited for aerospace and mobile communication. Their low power handling capability posits these antennas better in low power transmission and receiving applications [2]. The flexibility of the Microstrip antenna to shape it in multiple ways, like square, rectangular, circular, elliptical, triangular shapes etc., is an added property.

The rectangular and circular patches (Figure 1) are the basic and most commonly used designs in micros- trip antennas. Their designing methods are numerous, yet getting the actual data for developing real prototypes for experiment is found to be difficult. ANN offers a viable solution to obtain the design parameters. Hence, in this paper we have tried to develop the Quasi Newton algorithm for the design of circular patch antennas.

ANN is the most powerful optimizing tool in the field of computational electromagnetic. An ANN consists of interconnected processing units that store experimental knowledge. Such; this knowledge is acquired by a learning process and is stored in the form of parameters of the ANN [3]. The basic characteristics of ANN is its ability to learn and generalize, fault tolerance, non- linearity, and adaptivity.

The learning in ANN can be unsupervised or supervised. When an ANN undergoes learning in an unsupervised manner, it extracts the features from the input data based on a predetermined performance measure. When an ANN undergoes learning in a supervised manner, it is presented with the input patterns and the desired output patterns. The parameters of the ANN are adapted such that the application of an input pattern results in the desired pattern at the output of the ANN [4]. The Quasi Newton is one of the proven universal approximator in ANN design.

The design of CMSA as a closed form expression is given in Section 2. The Section 3 of the paper contains the description regarding the QN algorithm and design of the microstrip antenna as the analysis and synthesis model.

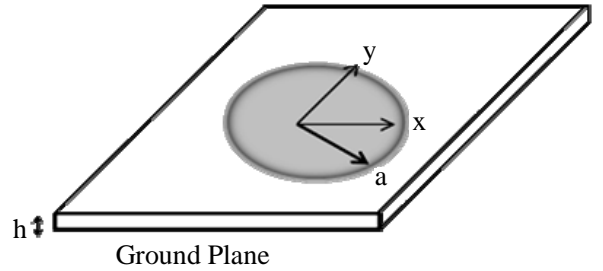

Figure 1. Circular microstrip antenna (CMSA) 
The results and conclusions are described in Section 4 and 5 respectively. ANN models are developed by using NeuroModeler 1.5 tool [5].

\section{A Closed Form Expression for CMSA Design}

The performance of circular microstrip antennas has been studied extensively, both analytically and experimentally. Consider the circular microstrip antenna with radius a, height $h$ and permittivity constant $\varepsilon_{r}$ whose resonant frequency in the dominant TM11 mode as explained by Guney [6], is given by (1).

And

$$
\varepsilon_{\text {eff }}=\frac{\varepsilon_{r}+1}{2}+\frac{\varepsilon_{r}-1}{2}\left[1+12 \frac{h}{a \sqrt{\pi}}\right]^{-1 / 2}
$$

where $\alpha_{n m}$ - the $m$ th zero of the derivatives of the Bessel function of order $n$

$c$ - the velocity of light.

$a$ - Radius of the circular patch

$f_{r}$ - resonant frequency of circular patch.

$h$ - Height of dielectric substrate

$\varepsilon_{r}$ - Permittivity of dielectric substrate

$$
\alpha_{n m}=1.84118 \mathrm{c} \quad(\text { for } n=m=1)
$$

The six patches of CMSA are designed by considering height $\mathrm{h}$ as 0.235 and $\varepsilon_{r}$ as 4.55 with variation of radius a.

The resonance frequency $f_{r}$ calculated by the Guney's equation was tested in four different and independent experiments. Their results when compared showed very little variation [7-10]. The deviation for resonant frequency of CMSA developed and measured by Abboud [7], Howell [8], Wolff [9] and Derneryd [10] is 0.19122, 0.0723,
$0.0788,0.238559$ respectively, which is very low as presented in Table 1. That explains the reason why the researchers chose Guney's equation for use in the present study of ANN modeling.

\section{ANN Modeling by Using QN Algorithm}

\subsection{QN Algorithm}

This is an advanced training algorithm in which second order derivative information is used. It approximates the inverse of the Hessian matrix of the training error function. The convergence of this method is almost similar to Newton's method when approaching the solution. The algorithm involves matrix operations. As such, computation effort becomes intensive as the size of the neural network increases. Hence, this training algorithm proves more useful for small and medium scale neural networks. The Quasi-Newton training may follow Conjugate Gradient to further reduce the training error. The formula under reference approximates the inverse Hessian matrix during optimization.

Here

$$
\Delta g=\nabla E(\text { epochs })-\nabla E(\text { epoch }-1)
$$

Quasi-Newton condition is:

$$
H \Delta w=\Delta g \text { or } \Delta w=B \Delta g
$$

where $H$ is Hessian Matrix,

$$
H=\frac{\partial}{\partial w}\left(\frac{\partial \boldsymbol{E}_{\boldsymbol{T}_{r}}}{\partial \boldsymbol{w}^{\boldsymbol{T}}}\right)
$$

$B$ is the approximation of inverse $H$.

Quasi-Newton method uses history of $\Delta w$ and $\Delta E$ to approximate $B$ :

$$
B(\text { epochs }=0)=I
$$

Table 1. Analysis of deviation of measured result from Guney’s equation

\begin{tabular}{ccccccccc}
\hline Fr (GHz) [6] & Abboud [7] & Deviation & Howell [8] & Deviation & Wolff [9] & Deviation & Derneryd [10] & Deviation \\
\hline 5.434885 & 4.945 & 0.489885 & 5.353 & 0.081885 & 5.308 & 0.126885 & 4.848 & 0.586885 \\
4.090526 & 3.750 & 0.340526 & 3.963 & 0.127526 & 3.950 & 0.140526 & 3.661 & 0.429526 \\
2.15468 & 2.003 & 0.15168 & 2.061 & 0.09368 & 2.067 & 0.08768 & 1.965 & 0.18968 \\
1.438828 & 1.360 & 0.078828 & 1.379 & 0.059828 & 1.384 & 0.054828 & 1.332 & 0.106828 \\
1.078434 & 1.030 & 0.048434 & 1.037 & 0.041434 & 1.042 & 0.036434 & 1.009 & 0.069434 \\
0.863001 & 0.825 & 0.038001 & 0.833 & 0.030001 & 0.836 & 0.027001 & 0.814 & 0.049001 \\
\multicolumn{2}{l}{ Average Deviation } & 0.1912256 & & 0.0723923 & & 0.0788923 & & 0.238559 \\
\hline
\end{tabular}

$$
f_{r}=\frac{1.84118 c}{2 \pi a\left[\varepsilon_{\text {eff }}\left\{1+\frac{2 h}{\pi \varepsilon_{r} a}\left(\ln \left(\frac{a}{2 h}\right)+\left(1.44 \varepsilon_{r}+1.77\right)+\frac{h}{a}\left(0.268 \varepsilon_{r}+1.65\right)\right)\right\}^{1 / 2}\right.}
$$




$$
\begin{aligned}
B(\text { epochs }+1)= & B(\text { epoch })+\frac{\Delta w \Delta w^{T}}{\Delta w^{T} \Delta g}\left(1+\frac{\Delta g^{T} B \Delta g}{\Delta w^{T} \Delta g}\right) \\
& -\frac{B \Delta g \Delta w^{T}+\Delta w \Delta g^{T} B}{\Delta g^{T} \Delta w}
\end{aligned}
$$

(BFGS Formula)

The speed of QN is high and the memory space required for it is $N 2 w$.

\subsection{Analysis Design of Circular Microstrip Antenna by Using QN Algorithm}

In this model, the accurate value of resonant frequency has been calculated by using Equation (1) and (2). The input parameters are permittivity $\varepsilon_{r}$, the height of substrate $h$ and patch dimension in terms of radius $a$.

The NN structure for the analysis of CMSA is shown in Figure 2. The Quasi-Newton algorithm has been considered for ANN modeling. The QN-ANN model consists of three layers i.e. input layer, hidden layer and output layer. The neurons for input layer are 3 and for hidden layer are 12; while the output layer has only one as shown in Figure 3. The epochs given for training the model were 200 from which it took only 80 epochs. For the purpose of training, 4397 data are generated for ANN modeling by application of Equation (1), from which 2199 data are selected while the remaining data are selected for testing the ANN model. The performance graph is displayed of training and testing in Figure $\mathbf{4}$ and Figure 5 respectively. As the experimental values of only 6 patches designed by [7-10] are given hence the result of only six patches is shown in Table 2. The output of ANN

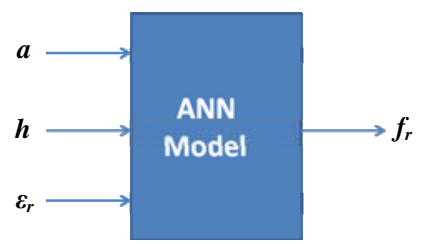

Figure 2. Analysis model of ANN

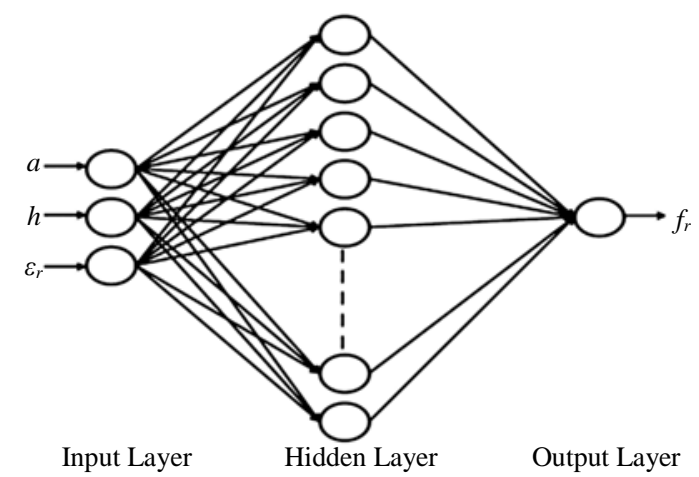

Figure 3. QN structure of analysis design of CMSA

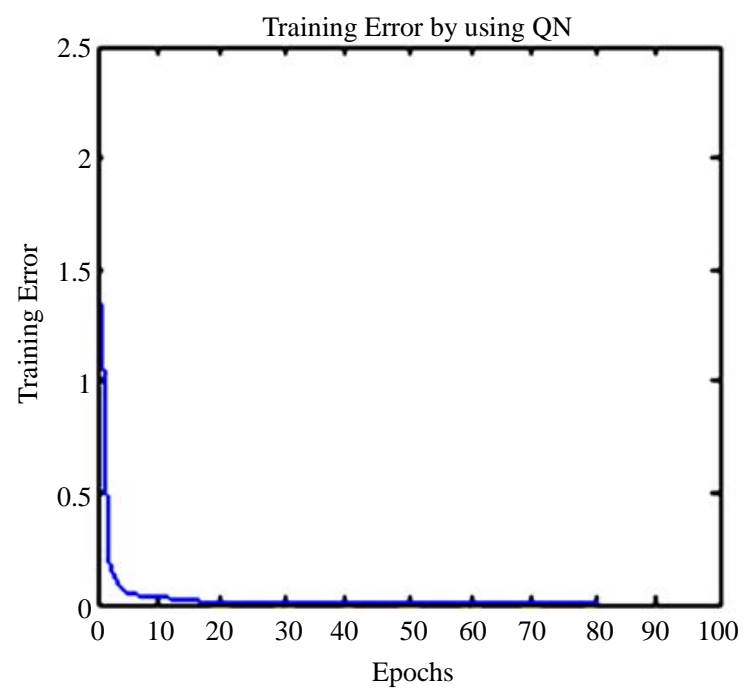

Figure 4. Performance result of QN algorithm developed for training analysis model of CMSA

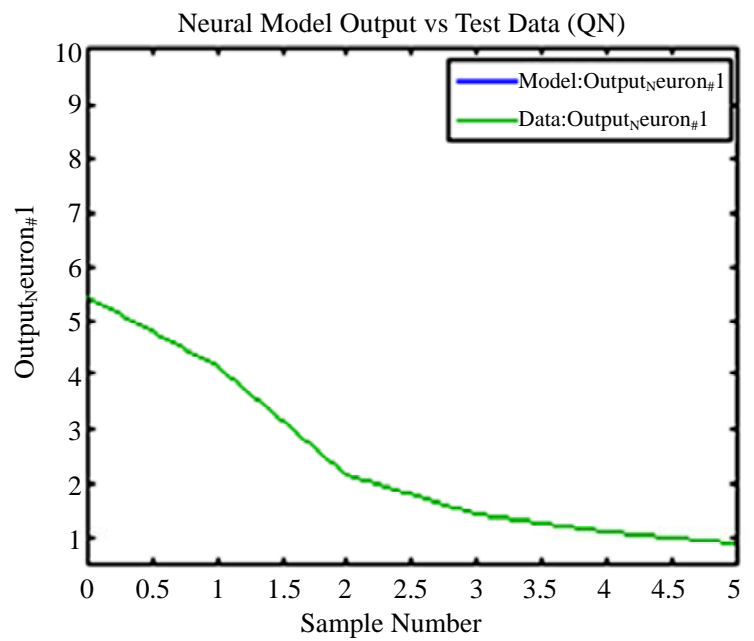

Figure 5. Performance result of testing QN-ANN model of CMSA

Table 2. Forward modeling for the Prediction of Resonant frequency

\begin{tabular}{cccccc}
\hline \multicolumn{3}{c}{ Input Parameters } & Target & \multicolumn{2}{c}{ QN } \\
\hline$a$ (radius) & $h$ & $\varepsilon_{r}$ & $f_{r}(\mathrm{GHz})$ & $\begin{array}{c}\text { ANN O/P } \\
(\mathrm{GHz})\end{array}$ & $\begin{array}{c}\text { Error } \\
(\mathrm{GHz})\end{array}$ \\
\hline 0.77 & 0.235 & 4.55 & 5.434885 & 5.441164 & 0.006278 \\
1.04 & 0.235 & 4.55 & 4.090526 & 4.086281 & -0.00425 \\
2 & 0.235 & 4.55 & 2.15468 & 2.154862 & 0.000182 \\
2.99 & 0.235 & 4.55 & 1.438828 & 1.439156 & 0.000328 \\
3.975 & 0.235 & 4.55 & 1.078434 & 1.078185 & -0.00025 \\
4.95 & 0.235 & 4.55 & 0.863001 & 0.866268 & 0.003267 \\
\multicolumn{2}{c}{ Average Value } & & 2.510059 & 2.510986 & 0.000926 \\
\hline \multicolumn{6}{c}{} \\
\end{tabular}


model is compared with the target data where QN-ANN model shows as little error as 0.000926, thereby laying a claim to be the best ANN analysis model for the design of CMSA.

\subsection{Synthesis Design of Circular Microstrip Antenna by Using QN Algorithm}

There are various methods available for the calculation of resonant frequencies of different patch antennas. But reverse calculation of radius from the inputs $f_{r}$, heigh $h$ and permitivitty constant $\varepsilon_{r}$ is not available in the literature. The solution for this is reverse modeling of ANN. The reverse model is also called as synthesis model which predicts the value of radius of circular patch as shown in Figure 6.The synthesis model consists of three layersinput layer with three neurons and hidden layer with 12 neurons and output layer with one neuron. The hidden layer uses sigmoid function while output layer uses linear function as shown in Figure 7.

The model is trained with 2199 data and training error graph is presented in Figure 8. The trained synthesis model is tested with 2198 data from which only six results are verified as its practically measured values are available in the literature [6-10] and it is given in Table 3. It takes only 101 epochs out of 200 for training synthesis model. The performance graph of testing synthesis model is presented in Figure 9. The actual error in values is given in Table 3 with very less error 0.0006 for the prediction of radius.

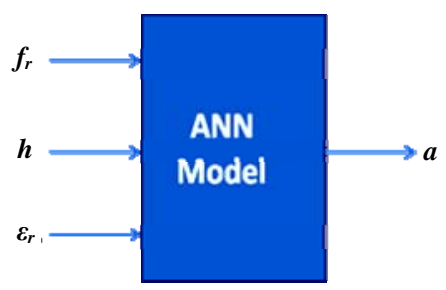

Figure 6. Synthesis model of ANN

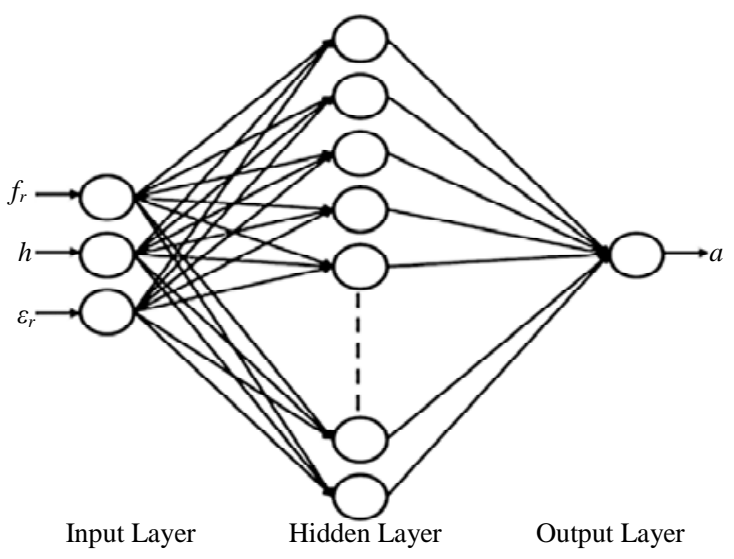

Figure 7. QN structure of synthesis design of CMSA

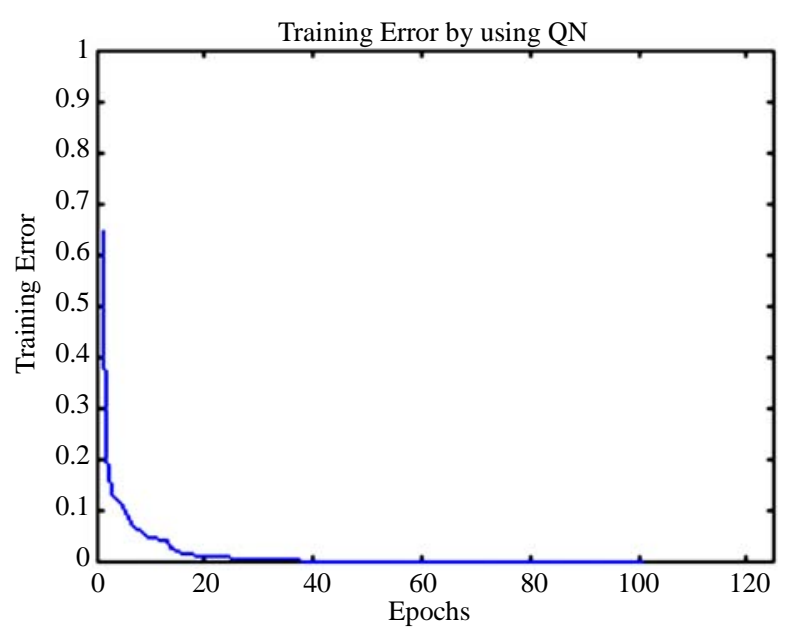

Figure 8. Performance result of QN algorithm developed for Training CMSA

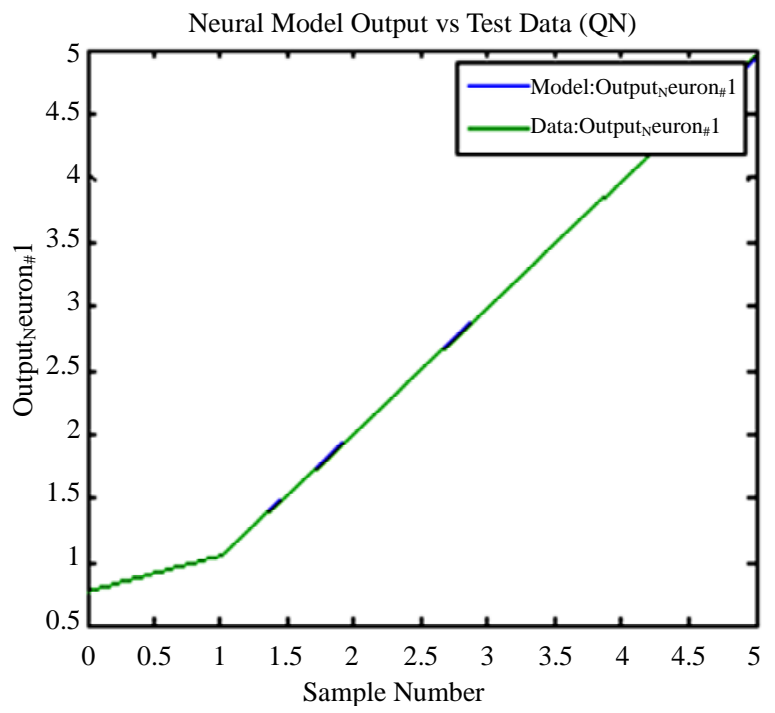

Figure 9. Performance result of testing QN-ANN model of CMSA

Table 3. Reverse modeling for the prediction of radius

\begin{tabular}{cccccc}
\hline \multicolumn{3}{c}{ Input Parameters } & Target & \multicolumn{2}{c}{ QN } \\
\hline$f_{r}(\mathrm{GHz})$ & $h$ & $\varepsilon_{r}$ & $a$ (radius) & $\begin{array}{c}\text { ANN O/P } \\
(\mathrm{GHz})\end{array}$ & $\begin{array}{c}\text { Error } \\
(\mathrm{GHz})\end{array}$ \\
\hline 5.434885 & 0.235 & 4.55 & 0.77 & 0.76673 & 0.00327 \\
4.090526 & 0.235 & 4.55 & 1.04 & 1.043208 & -0.00321 \\
2.15468 & 0.235 & 4.55 & 2 & 2.001889 & -0.00189 \\
1.438828 & 0.235 & 4.55 & 2.99 & 2.988124 & 0.001876 \\
1.078434 & 0.235 & 4.55 & 3.975 & 3.976177 & -0.00118 \\
0.863001 & 0.235 & 4.55 & 4.95 & 4.945272 & 0.004729 \\
\multicolumn{2}{c}{ Average Value } & & 2.620833 & 2.620233 & 0.0006 \\
\hline
\end{tabular}


Table 4. Analysis of computed, measured \& ANN predicted resonant frequencies

\begin{tabular}{ccccccccc}
\hline$a$ (radius) & $h$ & $\varepsilon_{r}$ & $f_{r}(\mathrm{GHz})$ & Abboud & Howell & Wolff & Derneryd & ANN Model \\
\hline 0.77 & 0.235 & 4.55 & 5.434885 & 4.945 & 5.353 & 5.308 & 4.848 & 5.441164 \\
1.04 & 0.235 & 4.55 & 4.090526 & 3.750 & 3.963 & 3.950 & 3.661 & 4.086281 \\
2 & 0.235 & 4.55 & 2.15468 & 2.003 & 2.061 & 2.067 & 1.965 & 2.154862 \\
2.99 & 0.235 & 4.55 & 1.438828 & 1.360 & 1.379 & 1.384 & 1.332 & 1.439156 \\
3.975 & 0.235 & 4.55 & 1.078434 & 1.030 & 1.037 & 1.042 & 1.009 & 1.078185 \\
4.95 & 0.235 & 4.55 & 0.863001 & 0.825 & 0.833 & 0.836 & 0.814 & 0.866268 \\
\multicolumn{2}{c}{ Average Deviation } & & & 0.1912256 & 0.0723923 & 0.0788923 & 0.238559 & 0.000926 \\
\hline
\end{tabular}

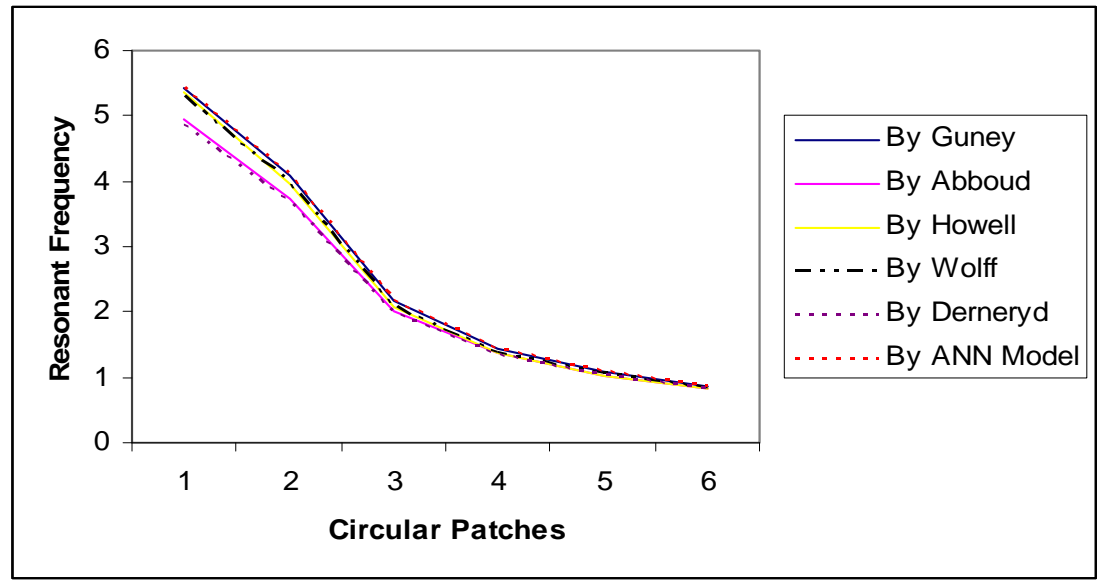

Figure 10. Comparison of measured $f_{r}$ by [6-10] and QN-ANN output

\section{Result}

The results demonstrate the excellent capacity of the neural model approximation which has been analyzed from Tables 1, 2, 3 and 4. The good agreement between the neural model answers and the fabricated patches as showed in Figure 10, demonstrates a good generalization capacity of the model through the Quasi-Newton modular structure. Besides, the QN structure, directly trained by means of measured/simulated data through the EMANN technique, is very flexible. It has the potential to be substituted, as models, mainly when new components/ technologies for microwaves circuits come up.

\section{Conclusions}

The neural models presented in this work have been found to possess high accuracy and requires no complicated mathematical functions. Using these models one can calculate resonant frequency of circular microstrip antenna accurately. The second ANN model of synthesis has unique characteristic of predicting radius of circular patches which is not available in the literature. If more data set is used for the training, the $\mathrm{NN}$ model gives more robust results. The analysis and synthesis models of ANN, gives better result for CMSA design which is found to compare well with the fabricated and measured values. So, it can be concluded that both the models are efficient for the prediction of resonant frequency and radius of the circular patch for all the practical purposes.

\section{Acknowledgements}

Authors acknowledge the support and valuable guidance; they have received from Prof. Q. J. Zhang, Professor and Chair, Department of Electronics, Carleton University, Ottawa, Canada in preparation of this paper.

\section{REFERENCES}

[1] G. Kumar and K. P. Ray, "Broadband Microstrip Antennas” Artech House, London, 2003.

[2] G. Garg, P. Bhartia, I. Bahl and A. Ittipiboon, "Microstrip Antenna Design Handbook,” Artech House, Canton, 2001.

[3] B. Yegnanarayana, “Artificial Neural Networks,” Prentice-Hall of India, Delhi, 1999.

[4] Q. J. Zhang, K. C. Gupta, and V. K. Devabhaktuni, “Artificial Neural Networks for R.F. and Microwave Design: from Theory to Practice,” IEEE Transactions on Microwave Theory and Techniques, Vol. 51, No. 4, April 2003, pp. 1339-1350. 
[5] Q. J. Zhang, “NeuroModeler Version 1.5 Software,” Carleton University, Ottawa, 2004.

[6] K. Guney, "Resonant Frequency of Electrically-Thick Circular Microstrip Antenna,” International Journal of Electronics, Vol. 77, No. 3, 1994, pp. 377-386.

[7] F. Abbound, J. P. Damiano and A. Papiernik, "New Determination of Resonant Frequency of Circular Disc Microstrip Antenna: Application to Thick Substrate,” Electronics Letters, Vol. 24, No. 17, 1988, pp. 1104-1106.

[8] J. Q. Howell, “Microstrip Antenna,” IEEE Transactions on Antennas and Propagation, Vol. 23, No. 1, 1975, pp. 90-93.

[9] I. Wolff and N. Knoppik, "Rectangular and Circular Microstrip Disk Capacitors and Resonators,” IEEE Transactions on Microwave Theory and Techniques, Vol. 22, No. 10, October 1974, pp. 857-864.

[10] A. G. Derneryd, "Microstrip Disc Antenna Covers Multiple Frequencies,” Microwave Journal, Vol. 21, May 1978, pp. 77-79. 